\title{
S-ROCK: CHEBYSHEV METHODS FOR STIFF STOCHASTIC DIFFERENTIAL EQUATIONS*
}

\author{
ASSYR ABDULLE ${ }^{\dagger}$ AND STEPHANE CIRILLI ${ }^{\ddagger}$
}

\begin{abstract}
We present and analyze a new class of numerical methods for the solution of stiff stochastic differential equations (SDEs). These methods, called S-ROCK (for stochastic orthogonal Runge-Kutta Chebyshev), are explicit and of strong order 1 and possess large stability domains in the mean-square sense. For mean-square stable stiff SDEs, they are much more efficient than the standard explicit methods proposed so far for stochastic problems and give significant speed improvement. The explicitness of the S-ROCK methods allows one to handle large systems without linear algebra problems usually encountered with implicit methods. Numerical results and comparisons with existing methods are reported.
\end{abstract}

Key words. stiff problems, stochastic differential equations, mean-square stability, Chebyshev methods, Runge-Kutta methods, explicit methods

AMS subject classifications. 60H10, 65C20, 65L20, 65N30

DOI. $10.1137 / 070679375$

1. Introduction. The importance of numerical methods for stochastic differential equations (SDEs) cannot be overemphasized as SDEs are used in the modeling of many biological, chemical, physical, and economical systems. In this paper we propose a new class of explicit numerical methods for the solution of stiff SDEs written in the Stratonovich form as ${ }^{1}$

$$
d Y=f(t, Y) d t+g(t, Y) \circ d W(t), \quad Y\left(t_{0}\right)=Y_{0},
$$

where $Y(t)$ is a random variable with value in $\mathbb{R}^{d}, f:[0, T] \times \mathbb{R}^{d} \rightarrow \mathbb{R}^{d}$ is called the drift function, $g:[0, T] \times \mathbb{R}^{d} \rightarrow \mathbb{R}^{d}$ is called the diffusion function, and $W(t)$ is a 1dimensional Wiener process, $t \geq 0$. We emphasize that our method is also applicable to SDEs with an $m$-dimensional Wiener process, however, with a possible reduction of the order of convergence. We will assume that both $f$ and $g$ are continuous and uniformly Lipschitz continuous with respect to the second variable and satisfy a linear growth condition and that $Y_{0}$ is independent of the Wiener process $W(t)$ and has a finite second order moment. Under these assumptions, the existence and uniqueness of a (mean-square-bounded) strong solution can be shown (see, for example, [26, sect. $5.2]$ for details).

As for deterministic ordinary differential equations (ODEs), stiffness is a central issue for the numerical treatment of SDEs. Stiffness is concerned with (local) proper-

${ }^{*}$ Received by the editors January 6, 2007; accepted for publication (in revised form) October 2, 2007; published electronically March 5, 2008.

http://www.siam.org/journals/sisc/30-2/67937.html

†School of Mathematics and Maxwell Institute for Mathematical Sciences, University of Edinburgh, Edinburgh EH9 3JZ, UK (a.abdulle@ed.ac.uk). This work is partially supported by EPSRC Advanced Fellowship EP/E05207X/1 and a visit to the University of Geneva supported by the Swiss National Foundation grant 200020-113249/1.

‡Section de Mathématiques, Université de Genève, 2-4 rue de Lièvre, CH-1211 Genève 24, Switzerland (stephane.cirilli@unige.ch).

${ }^{1}$ The Stratonovich integral is chosen here for its "ordinary chain rule" property. Recall that any Itô stochastic differential equations can be converted in Stratonovich form. Thus, the numerical methods proposed in this paper apply to general SDEs.

997 
ties of the SDE which can affect the stability of a numerical method. For ODEs, it is well known that stiffness leads to step size restrictions when using traditional explicit solvers, and implicit methods are usually advocated for such problems [15]. The good stability properties of implicit methods are obtained at the cost of solving nonlinear equations at each step. Although efficient in many situations, this approach can be expensive especially for large systems. The development of explicit methods for the numerical solution of stiff ODEs has been considered by many researchers. Successful methods, so-called Chebyshev methods, have been constructed for dissipative problems [1],[2],[19],[21],[30]. Other types of explicit methods have been proposed for stiff problems with scale separation [9],[11],[13].

For SDEs, one can consider three classes of methods: explicit methods, semiimplicit methods (implicit only in the drift term), and fully implicit methods. The latter methods have to be considered with care due to the possible unboundedness of the solution [6],[25]. Such fully implicit methods have been constructed for stiff problems where some components of a stiff multidimensional system have a small or vanishing drift term for which semi-implicit methods cannot improve the stability of the numerical solution [25].

In this paper, we focus on mean-square stable SDEs (to be defined below), and for such problems semi-implicit methods are applicable [18],[17]. However, as mentioned above, due to the implicitness and linear algebra problems, such methods can be costly. Explicit methods for stochastic problems with multiple scales which can be separated into fast and slow components have been proposed in [31],[10]. For stiff stochastic problems without scale separation, efficient explicit methods for stiff meansquare stable problems have not been considered to the best of the authors' knowledge. We propose here a new class of explicit methods of strong order 1 with large stability domains in the mean-square sense called S-ROCK (for stochastic orthogonal RungeKutta Chebyshev methods) first introduced in [8]. ROCK methods are a class of second and fourth order explicit Chebyshev methods based on orthogonal stability functions introduced in [1] and [2], respectively, for the efficient numerical solution of stiff ODEs. The S-ROCK methods discussed in this paper extend Chebyshev methods to stiff SDEs. Due to their explicitness, they are as easy to implement as standard explicit methods for SDEs (as, for example, the well-known Euler-Maruyama method). At the same time, due to their extended stability properties, these methods are well suited for stiff mean-square stable SDEs.

The paper is organized as follows. In section 2 we recall the convergence and stability concepts that we need in what follows. In section 3 we introduce the S-ROCK methods discuss the order and the stability of these methods. At the end of section 3 the general case of SDEs with multidimensional Wiener processes is discussed. Finally, in section 4 we present numerical examples and comparison with other methods to illustrate the behavior of the S-ROCK methods.

2. Stability and convergence for stochastic methods. We consider onestep methods for the numerical solution of the SDE (1.1) of the form

$$
Y_{n+1}=\Phi\left(Y_{n}, \Delta t, J_{1, n}, \ldots, J_{l, n}\right),
$$

where $J_{j, n}=W_{j}\left(t_{n+1}\right)-W_{j}\left(t_{n}\right)$ and $W_{j}(t)$ is a Wiener process. The increment $W_{j}(t)-W_{j}(s)$ is normally distributed with mean zero and variance $t-s$, and $J_{j, n}$ can be evaluated by computing $\sqrt{\Delta t} \mathcal{N}_{j}\left(\mathcal{N}_{j}\right.$ are independent standard Gaussian random vectors with distribution $\mathcal{N}(0,1))$. 
An early method introduced for SDEs is the so-called Euler-Maruyama method [22] given $b^{2}$

$$
Y_{n+1}=Y_{n}+\Delta t f\left(Y_{n}\right)+J_{n} g\left(Y_{n}\right)
$$

This method has a low order of convergence, and several authors [23], [27] proposed higher order methods (in a sense to be defined below). As an example, we consider the method introduced by Platen [27], given by a two-stage scheme

$$
\begin{array}{r}
K_{n}=Y_{n}+\Delta t f\left(Y_{n}\right)+J_{n} g\left(Y_{n}\right), \\
Y_{n+1}=Y_{n}+\Delta t f\left(Y_{n}\right)+J_{n} \frac{1}{2}\left(g\left(Y_{n}\right)+g\left(K_{n}\right)\right) .
\end{array}
$$

We recall the concepts of accuracy and stability for the numerical integration of SDEs. A method is said to have a strong order $\rho$ (respectively, weak order of $v$ ) if there exists a constant $C$ such that

$$
\mathbb{E}\left(\left|Y_{N}-Y(\tau)\right|\right) \leq C \Delta t^{\rho} \text { (strong), } \mid \mathbb{E}\left(h\left(Y_{N}\right)\right)-\mathbb{E}\left(h(Y(\tau)) \mid \leq C \Delta t^{v}\right. \text { (weak) }
$$

for any fixed $\tau=N \Delta t \in[0, T]$ and $\Delta t$ sufficiently small and for all functions $h: \mathbb{R}^{d} \rightarrow$ $\mathbb{R}, 2(v+1)$ times continuously differentiable and for which all partial derivatives have polynomial growth.

Remark 2.1. Note that, for every function $h$ satisfying a Lipschitz condition, $\left|\mathbb{E}\left(Y_{N}\right)-\mathbb{E}(Y(\tau))\right| \leq C \Delta t^{\rho}$ implies $\left|\mathbb{E}\left(h\left(Y_{N}\right)\right)-\mathbb{E}(h(Y(\tau)))\right| \leq C \Delta t^{\rho}$, and, thus, strong global convergence of order $\rho$ implies weak global convergence of order $\rho$. However, the weak global order obtained in this manner may not be optimal.

It is useful for numerical purposes to have a relationship between the global convergence as described in (2.5) and the local convergence, i.e., the convergence after one step when the initial solution for the numerical method (2.1) is taken on the exact solution. This relation given by Milstein is as follows (see [24],[5]).

TheOREM 2.2 (Milstein 1987). Suppose that $g$ and $f$, the drift and diffusion, respectively, of the $S D E(1.1)$, are continuous on $[0, T] \times \mathbb{R}^{d}$ and sufficiently smooth and satisfy a uniform Lipschitz condition

$$
|f(t, y)-f(t, z)|+|g(t, y)-g(t, z)| \leq L|y-z| \forall t \in[0, T], y, z \in \mathbb{R}^{d},
$$

and suppose further that the one-step method (2.1) satisfies the following local order conditions:

$$
\begin{aligned}
& \mathbb{E}\left(\left|Y_{1}-Y\left(t_{0}+\Delta t\right)\right|\right) \leq C \Delta t^{\rho+1 / 2}, \\
& \left|\mathbb{E}\left(Y_{1}\right)-\mathbb{E}\left(Y\left(t_{0}+\Delta t\right)\right)\right| \leq C \Delta t^{\rho+1},
\end{aligned}
$$

and then the method converges with a strong global order $\rho$.

Remark 2.3. For methods with integer local strong order and which depend only on 1 Wiener increment $J_{1}$, the condition (2.8) will automatically be satisfied if (2.7) holds, since the expectation of any stochastic integral involving an odd number of Wiener increments will vanish (see [20],[5] for more details).

Going back to our two examples, it can be shown, by comparing the Taylor series expansion of the numerical methods and the true solution (see [20]), that the Platen method has strong order 1 and that the Euler-Maruyama method does not converge

\footnotetext{
${ }^{2}$ When working with one Wiener increment $J_{1, n}$, we will write it simply as $J_{n}$.
} 
towards the Stratonovich SDE (1.1) (it converges to the solution of the Itô problem $d Y=f(t, Y) d t+g(t, Y) d W(t))$.

The above convergence concepts concern the convergence of a numerical method for small step sizes $\Delta t \rightarrow 0$. For many applications, the efficiency of a numerical method over a long time interval will also depend on its stability properties. Widely used measures of stability for SDEs are mean-square stability, which measures the stability of moments, and asymptotic stability (in the large), which measures the overall behavior of sample functions [16]. In this paper we focus on the first stability concept. For a linear autonomous system of SDEs the former concept is stronger than the latter (see [4, Chap. 11]) or [16]). We notice that asymptotic stability of numerical methods has been studied recently by several authors [18],[28].

Consider the $\operatorname{SDE}(1.1)$ with $f(t, 0)=g(t, 0)=0$ and with a nonrandom initial value $Y_{0}$. The steady solution $Y \equiv 0$ is said to be mean-square stable if there exists $\delta_{0}$ such that

$$
\lim _{t \rightarrow \infty} \mathbb{E}\left(|Y(t)|^{2}\right)=0 \text { for all }\left|Y_{0}\right|<\delta_{0} .
$$

As for deterministic ODEs, where a large body of stability studies is based on the linear test equation $y^{\prime}=\lambda y$, a lot of insight into the behavior of numerical methods for stochastic problems can be gained by studying the stochastic test problem [29],[18]

$$
d Y=\lambda Y d t+\mu Y \circ d W(t), \quad Y\left(t_{0}\right)=Y_{0},
$$

where $\lambda, \mu \in \mathbb{C}$. The solution of (2.10), $Y(t)=Y_{0} \exp (\lambda t+\mu W(t))$, is mean-square stable if and only if $\Re \lambda+\Re \mu^{2}<0$ (see, for example, [4],[26]).

We denote the stability region of the test problem by

$$
\mathcal{S}_{S D E}:=\left\{\lambda, \mu \in \mathbb{C} ; \Re \lambda+\Re \mu^{2}<0\right\} .
$$

Following [29] we will say that a one-step method (2.1) is mean-square stable (for the problem (2.10)) if

$$
\lim _{n \rightarrow \infty} \mathbb{E}\left(\left|Y_{n}\right|^{2}\right)=0
$$

Applying the Euler-Maruyama method to the above linear test equation produces

$$
Y_{n+1}=\left(1+\Delta t \lambda+J_{n} \mu\right) Y_{n}
$$

Squaring the modulus of (2.13), taking the expected value, and using $\mathbb{E}\left(J_{n}\right)=0$, $\mathbb{E}\left(J_{n}^{2}\right)=\Delta t$ gives $\mathbb{E}\left(\left|Y_{n+1}\right|^{2}\right)=\left(|1+p|^{2}+|q|^{2}\right) \mathbb{E}\left(\left|Y_{n}\right|^{2}\right)$, where $p=\Delta t \lambda, q=\sqrt{\Delta t} \mu$. Thus, the Euler-Maruyama method is mean-square stable if and only if $R(p, q)=\mid 1+$ $\left.p\right|^{2}+|q|^{2}<1$. A similar treatment can be done for the Platen method. More generally, a one-step method (2.1) applied to the test equation (2.10) typically produces

$$
\mathbb{E}\left(\left|Y_{n+1}\right|^{2}\right)=R(p, q) \mathbb{E}\left(\left|Y_{n}\right|^{2}\right),
$$

where $R(p, q) \geq 0$ and the method is seen to be mean-square stable for the problem (2.10) if and only if $R(p, q)<1$. The stability domain of a numerical method can thus be defined as

$$
\mathcal{S}:=\{p, q \in \mathbb{C} ; R(p, q)<1\}
$$



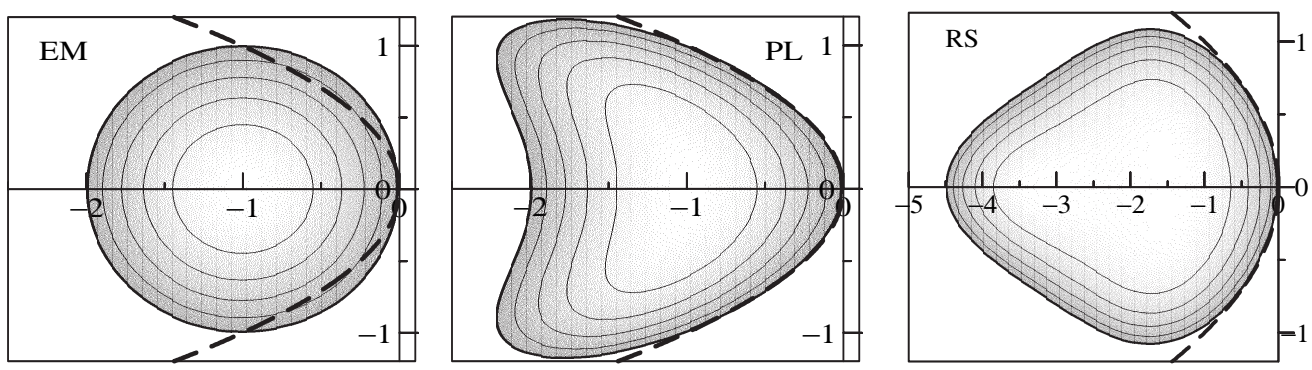

FIG. 2.1. Mean-square stability domains for the Euler-Maruyama (EM) method, the Platen (PL) method, and the RS method.

the sets of pairs of complex numbers $p, q$ for which the method is (mean-square) stable. In Figure 2.1 we plotted the stability domain of the Euler-Maruyama (EM) and Platen (PL) methods for $\lambda, \mu \in \mathbb{R}$. We also plotted the stability domain of the so-called RS method [7, p. 187], a two-stage method constructed with the aim of improving its stability property. We will further discuss this method in section 4 . As a dashed line we sketched the boundary of the domain for which the test equation is mean-square stable (the left part of the parabola is the stable region). ${ }^{3}$ We see that the three methods cover only a small part of the stability region of the exact solution. Thus, for stiff problems (e.g., $\lambda, \mu$ large) very small step sizes $\Delta t$ are required for a stable numerical solution. Notice that the stability region of the RS method along the $p$-axis is twice as big as the corresponding region of the EM or PL methods.

In the next section we will construct explicit methods with stability domains covering a much larger region of the left part of the dashed parabola (the true stability region).

\section{S-ROCK: Stochastic Chebyshev methods.}

3.1. Chebyshev methods for deterministic problems. Chebyshev methods (which will be generalized with the S-ROCK methods for stochastic problems) are a class of explicit one-step methods with extended stability domains along the negative real axis. The basic idea for such methods goes back to the 1960s with Saul'ev, Franklin, and Guillou and Lago (see [15, sect. IV.2] and the references therein) and is as follows: Consider a sequence of forward Euler methods $\Psi_{h_{1}}, \ldots, \Psi_{h_{m}}$ with a corresponding sequence of time steps $h_{1}, \ldots, h_{m}$, and define a one-step method as the composition $\Psi_{\Delta t}=\left(\Psi_{h_{m}} \circ \cdots \circ \Psi_{h_{1}}\right)\left(y_{0}\right)$ with step size $\Delta t=h_{1}+\cdots+h_{m}$. Next, given $m$, optimize the sequence $\left\{h_{i}\right\}_{i=1}^{m}$ so that

$$
\left|P_{m}(x)\right|=\left|\prod_{i=1}^{m}\left(1+h_{i} x\right)\right| \leq 1 \text { for } x \in\left[-l_{m}, 0\right],
$$

with $l_{m}>0$ as large as possible. The solution of this problem is given by shifted Chebyshev polynomials

$$
\begin{aligned}
& P_{m}(x)=T_{m}\left(1+x / m^{2}\right), \\
& T_{0}(x)=1, T_{1}(x)=x, \quad T_{j}(x)=2 x T_{j-1}(x)-T_{j-2}(x), j \geq 2
\end{aligned}
$$

\footnotetext{
${ }^{3}$ We notice that some authors use the scaling $(p, q)=\left(\Delta t \lambda, \Delta t \mu^{2}\right)$ for stability region diagrams for which the stability region of the test problem becomes a wedge [29], [18].
} 


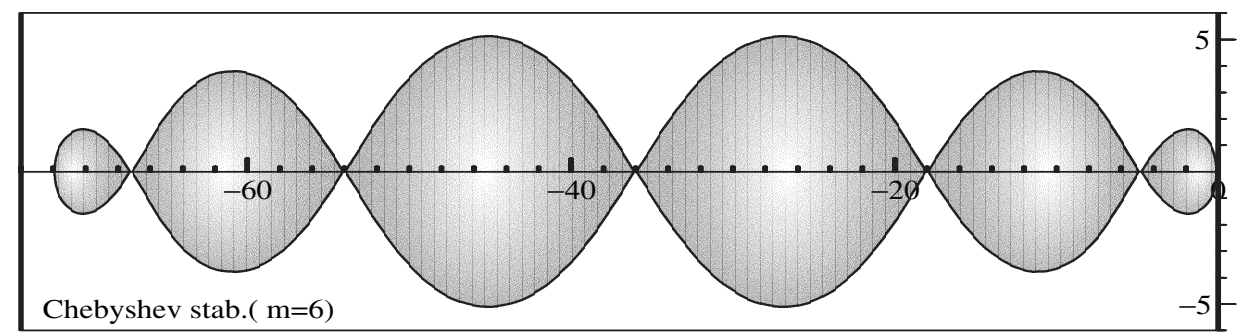

FIG. 3.1. Stability region for shifted Chebyshev polynomials of degree $m=6$.

i.e., the optimal sequence of $\left\{h_{i}\right\}_{i=1}^{m}$ is given by $h_{i}=-1 / x_{i}$, where $x_{i}$ are the zeros of $P_{m}(x)$, and the maximal stability domain on the negative real axis increases quadratically with the number of stages and is given by $l_{m}=2 m^{2}$ (see Figure 3.1). Recall that $m$, the number of stages of the method, represents the numerical work per step (functions evaluation). Thus, if for the stable numerical integration of an initial value problem from $t_{0}=0$ to $t_{m}=T, N$ functions evaluation is needed for the forward Euler method, the above Chebyshev method will need only $\sqrt{N}$ functions evaluation.

If one takes an arbitrary order of the sequence of step size $h_{1}, \ldots, h_{s}$, internal instability within a single integration step $\Delta t$ can occur, and the numerical method will be useless. To overcome this problem, one can either use a special ordering of the sequence of steps as suggested in [21] or use the three-term recurrence relation of the Chebyshev polynomial as suggested in [19]. Following [19], we consider a $m$-stage numerical method given by

$$
\begin{aligned}
k_{0} & :=y_{0}, \\
k_{1} & :=y_{0}+\frac{\Delta t}{m^{2}} f\left(k_{0}\right), \\
k_{j} & :=\frac{2 \Delta t}{m^{2}} f\left(k_{j-1}\right)+2 k_{j-1}-k_{j-2}, \\
y_{1} & :=k_{m} .
\end{aligned}
$$

Applied to the test problem $y^{\prime}=\lambda y$, this method gives for the internal stages

$$
k_{j}=T_{j}\left(1+\Delta t \lambda / m^{2}\right) y_{0}, j=0, \ldots, m,
$$

and produces after one step $y_{1}=R_{m}(\Delta t \lambda) y_{0}$, where $R_{m}(x)=T_{m}\left(1+x / m^{2}\right)$ is a polynomial of degree $m . R_{m}(x)$ is called the stability function of the numerical method. Since $R_{m}(x)=1+x+\mathcal{O}\left(x^{2}\right)$, the method is of order one; i.e., the global error after $n$ steps is $\mathcal{O}(\Delta t)$ for all sufficiently smooth (deterministic) initial value problems. A method of order 2 based on a linear combination of Chebyshev polynomials has been given in [19],[30], and the ROCK methods of order 2 and 4 based on quasioptimal orthogonal polynomials combining the approaches of [30] and [21] have been proposed in [1],[2].

It was already observed by Guillou and Lago [14] that the stability requirement $\left|R_{m}(z)\right| \leq 1, z \in \mathbb{C}$ (weakly stable polynomials), should be replaced by $\left|R_{m}(z)\right| \leq$ $q<1$ (strongly stable polynomials). Indeed, for the points $x_{i} \in \mathbb{R}^{-}$, where $R\left(x_{i}\right)=$ $T_{m}\left(1+x_{i} / m^{2}\right)= \pm 1$, the stability domain has zero width (see Figure 3.1). If one sets

$$
R_{m}(z)=\frac{1}{T_{m}\left(\omega_{0}\right)} T_{m}\left(\omega_{0}+\omega_{1} z\right), \quad \omega_{0}=1+\frac{\eta}{m^{2}}, \quad \omega_{1}=\frac{T_{m}\left(\omega_{0}\right)}{T_{m}^{\prime}\left(\omega_{0}\right)},
$$




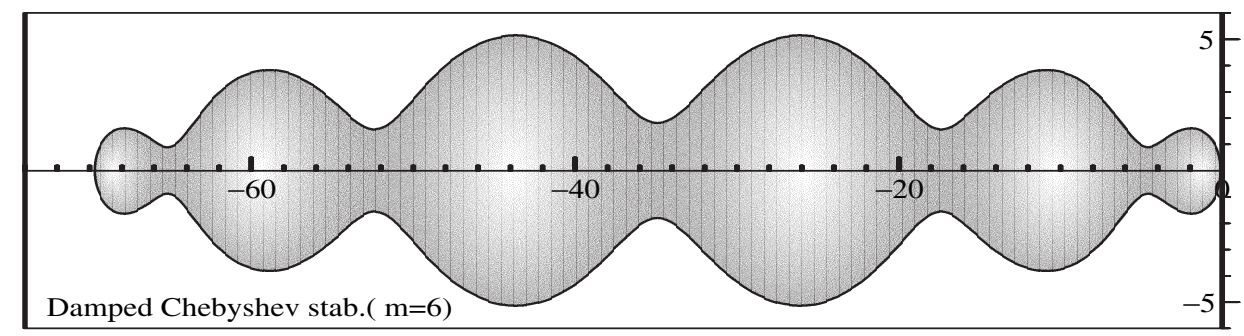

FIG. 3.2. Stability region for shifted Chebyshev polynomials of degree $m=6$ with damping.

then the above polynomials oscillate approximately between $-1+\eta$ and $1-\eta$. The stability domain along the negative real axis is a bit shorter, but the damping ensures that a strip around the negative real axis is included in the stability domain (see Figure 3.2). A modification of the method (3.2) allows one to construct a Chebyshev method with damping. All of the methods proposed in [1],[2],[19],[21],[30] are realized with damping.

3.2. S-ROCK: Chebyshev methods for stochastic problems. In this section we introduce our new stochastic methods. We know that, for deterministic problems, Chebyshev methods can increase their stability domains quadratically (along the negative real axis) with the number of stages (see section 3.1). The idea here is to use the aforementioned properties of the Chebyshev polynomials to handle the stiffness of the problem and to incorporate the Wiener process in a two-stage finishing procedure similarly as high order is realized with the ROCK methods (see [1],[2]).

In order to simplify the presentation we consider (1.1) in autonomous form ${ }^{4}$

$$
d Y=f(Y) d t+g(Y) \circ d W(t), \quad Y\left(t_{0}\right)=Y_{0}, Y(t) \in \mathbb{R}^{d} .
$$

To motivate the methods, we consider (3.2) with $m=2$ and incorporate Wiener increments in the following way:

$$
\begin{aligned}
& K_{0}:=Y_{n}, \\
& K_{1}:=Y_{n}+\frac{\Delta t}{4} f\left(K_{0}\right)+J_{n} \alpha g\left(K_{0}\right), \\
& K_{2}:=\frac{\Delta t}{2} f\left(K_{1}\right)+2 K_{1}-K_{0}+J_{n}\left(\beta g\left(K_{0}\right)+\gamma g\left(K_{1}\right)\right), \\
& Y_{n+1}:=K_{2},
\end{aligned}
$$

where $\alpha, \beta$, and $\gamma$ are real parameters and $J_{n}=W\left(t_{n+1}\right)-W\left(t_{n}\right)$. The Taylor series expansion of this numerical method is given (up to first order terms) by

$$
Y_{1}=Y_{0}+J_{0} f_{0}+J_{1}(2 \alpha+\beta+\gamma) g_{0}+J_{1}^{2} \alpha \gamma\left(g^{\prime} g\right)_{0}+R_{1},
$$

where $R_{1}$ contains higher order terms. In this formula we used the notation $J_{0}=$ $\int_{t_{0}}^{t} d s_{1}, J_{1}=\int_{t_{0}}^{t} \circ d W_{s_{1}}$. For the exact solution of (3.5) we obtain the expansion

$$
Y(t)=Y_{0}+J_{0} f_{0}+J_{1} g_{0}+J_{11}\left(g^{\prime} g\right)_{0}+R_{e},
$$

\footnotetext{
${ }^{4} \mathrm{An}$ SDE can always be written in autonomous form by adding another component representing the time.
} 

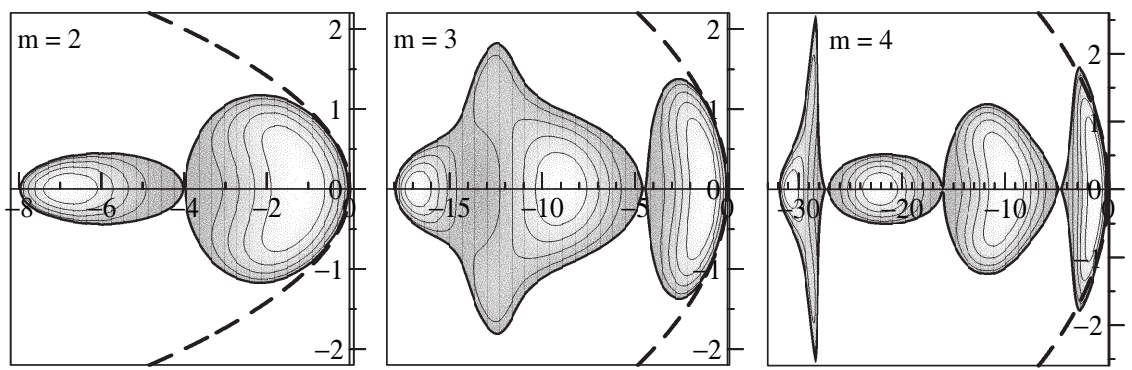

FIG. 3.3. Mean-square stability domains for the stochastic ROCK methods with $m=2,3$, and 4 stages.

where $J_{11}=\int_{t_{0}}^{t_{1}} \int_{t_{0}}^{s_{1}} \circ d W_{s_{2}} \circ d W_{s_{1}}$. By comparing the two expansions and using $J_{1}^{2}=$ $2 J_{11}$ we find that this method will be of (local) strong order 1 if and only if

$$
2 \alpha+\beta+\gamma=1 \quad \text { and } \quad \alpha \gamma=\frac{1}{2} .
$$

By Theorem 2.2 and Remark 2.3 it follows that the method will also be of global strong order 1 if and only if conditions (3.9) are satisfied.

In (3.9) we have a degree of liberty. We choose $\alpha$ as a free parameter, optimize its value in order to maximize the mean-square stability region, and find $\alpha=\frac{1}{2}, \beta=-1$, and $\gamma=1$. For these values and for $\lambda, \mu$ real, we obtain the mean-square stability function

$$
R_{2}(p, q)=1+2\left(p+q^{2}\right)+\frac{5}{4} p^{2}+2 p q^{2}+\frac{3}{4} q^{4}+\frac{1}{4} p^{3}+\frac{3}{8} p^{2} q^{2}+\frac{1}{64} p^{4} .
$$

The stability domain (see Definition 2.15) of the above method is drawn in the left frame of Figure 3.3. Recall that $p=\Delta t \lambda$ and $q=\sqrt{\Delta} t \mu$. Here and in what follows, we will plot the stability region in the real $(p, q)$ plane, where on the horizontal axis we plot the $p$ variable and on the vertical axis we plot the $q$ variable. We observe in Figure 3.3 (left frame) that the stability domain contains the interval $\left[-l_{2}, 0\right]$, where $l_{2}=8$. The value $l_{2}$ is the maximum value on the (real negative) $p$-axis that an explicit two-stage stochastic numerical method can reach. This follows from the optimality of the Chebyshev polynomial $T_{2}\left(1+x / s^{2}\right)$ (see section 3.1). Although the stability domain is optimal on the $p$-axis (compare with Figure 1), this is not satisfactory since the width of the stability domain in the $q$ direction vanishes in some regions. This drawback will be overcome later by using damping techniques. We first generalized the method (3.6) for arbitrary stages and consider for $m>2$ the $m$-stage stochastic ROCK methods defined by

$$
\begin{aligned}
& K_{0}=Y_{n}, \\
& K_{1}=Y_{n}+\frac{\Delta t}{m^{2}} f\left(K_{0}\right), \\
& K_{i}=\frac{2 \Delta t}{m^{2}} f\left(K_{i-1}\right)+2 K_{i-1}-K_{i-2}, \quad i=2, \ldots, m-2, \\
& K_{m-1}=\frac{2 \Delta t}{m^{2}} f\left(K_{m-2}\right)+2 K_{m-2}-K_{m-3}+J_{n} \alpha g\left(K_{m-2}\right), \\
& Y_{n+1}=K_{m}=\frac{2 \Delta t}{m^{2}} f\left(K_{m-1}\right)+2 K_{m-1}-K_{m-2}+J_{n}\left(\beta g\left(K_{m-2}\right)+\gamma g\left(K_{m-1}\right)\right) .
\end{aligned}
$$


We observe that, for $g \equiv 0$, these methods are identical to the deterministic methods (3.2).

THEOREM 3.1. For $m \geq 2$, the methods (3.11) have strong global order one if and only if

$$
2 \alpha+\beta+\gamma=1 \quad \text { and } \quad \alpha \gamma=\frac{1}{2} .
$$

The mean-square stability function is given, for $\lambda$ and $\mu$ real, by

$$
\begin{aligned}
R_{m}(p, q)= & T_{m}^{2}\left(1+\frac{p}{m^{2}}\right)+q^{2}\left(\gamma T_{m-1}\left(1+\frac{p}{m^{2}}\right)+\left(\frac{2 p \alpha}{m^{2}}+2 \alpha+\beta\right) T_{m-2}\left(1+\frac{p}{m^{2}}\right)\right)^{2} \\
& +(\alpha \gamma)^{2} q^{2} T_{m}\left(1+\frac{p}{m^{2}}\right) T_{m-2}\left(1+\frac{p}{m^{2}}\right)+\frac{3}{4}(\alpha \gamma)^{2} q^{4} T_{m-2}^{2}\left(1+\frac{p}{m^{2}}\right) .
\end{aligned}
$$

Proof. Expanding the deterministic stages of (3.11) in Taylor series gives by induction for $1 \leq j \leq m-2$

$$
K_{j}=Y_{n}+\Delta t \frac{a_{j}}{m^{2}} f\left(Y_{n}\right)+\mathcal{O}\left((\Delta t)^{2}\right)
$$

where the coefficients $a_{j}$ satisfy the recurrence $a_{j}=2+2 a_{j-1}-a_{j-1}, a_{1}=1, a_{0}=0$. For the last two stages similarly as in (3.7) we have

$$
\begin{aligned}
K_{m-1} & =Y_{n}+\Delta t \frac{a_{m-1}}{m^{2}} f\left(Y_{n}\right)+J_{n} \alpha g\left(Y_{n}\right)+\mathcal{O}\left((\Delta t) J_{n}\right), \\
K_{m} & =\Delta t \frac{a_{m}}{m^{2}} f\left(Y_{n}\right)+J_{n}(2 \alpha+\beta+\gamma) g\left(Y_{n}\right)+J_{n}^{2} \alpha \gamma g^{\prime}\left(Y_{n}\right) g\left(Y_{n}\right)+\mathcal{O}\left((\Delta t)^{3 / 2}\right) .
\end{aligned}
$$

By induction we verify that $a_{j}=j^{2}$. Similarly as for the previous two-stage method, by comparing the above expansion and the expansion for the exact solution (3.8) and invoking Theorem 2.2 and Remark 2.3, we see that the methods (3.11) have strong global order one if and only if (3.12) holds. For the mean-square stability function, we apply the method (3.11) to the test problem (2.10), use the recurrence relation of the Chebyshev polynomial for the deterministic stages $j=1, \ldots, s-2$, and obtain $K_{m-2}=T_{m-2}\left(1+\frac{p}{m^{2}}\right) Y_{n}$, where $p=\Delta t \lambda$, and for the last two stages

$$
\begin{aligned}
K_{m-1}= & \left(T_{m-1}\left(1+\frac{p}{m^{2}}\right)+\alpha \mu T_{m-2}\left(1+\frac{p}{m^{2}}\right) J_{n}\right) Y_{n} \\
K_{m}= & \left(T_{m}\left(1+\frac{p}{m^{2}}\right)+\mu\left(\gamma T_{m-1}\left(1+\frac{p}{m^{2}}\right)+\left(\frac{2 p \alpha}{m^{2}}+2 \alpha+\beta\right) T_{m-2}\left(1+\frac{p}{m^{2}}\right)\right) J_{n}\right. \\
& \left.\quad+\alpha \gamma \mu^{2} T_{m-2}\left(1+\frac{p}{m^{2}}\right) J_{n}^{2}\right) Y_{n}
\end{aligned}
$$

where $p=\Delta t \lambda$. Squaring and taking the expectation, using $\mathbb{E}\left(J_{n}\right)=0, \mathbb{E}\left(J_{n}^{2}\right)=\Delta t$, $\mathbb{E}\left(J_{n}^{3}\right)=0$, and $\mathbb{E}\left(J_{n}^{4}\right)=3 \Delta t$, and setting $q=\sqrt{\Delta t} \mu$ gives (3.13).

As for the two-stage method (3.6), we chose the parameters $\alpha=1 / 2, \beta=-1, \gamma=$ 1. In Figure 3.4 we plotted the mean-square stability (MS) domain of the method (3.11) for different stage numbers $m$. We see that for $m=2,3,4$ these methods have a strip $\left[-l_{m}, 0\right]$ included in their stability domain $\mathcal{S}_{m}$, with $l_{m}=2 m^{2}$. This is true 

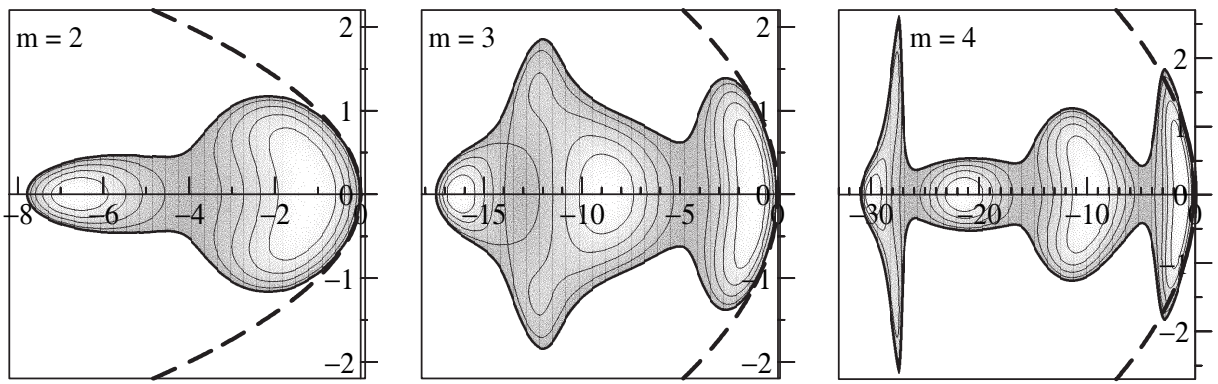

FIG. 3.4. MS domain for the stochastic ROCK methods with $m=2,3,4$ and $\eta=0.05$.

in general. Setting $q=0$, the mean-square stability condition for the method (3.11) reduces to $T_{m}\left(1+\frac{p}{m^{2}}\right)^{2} \leq 1$, the stability condition for the deterministic method (3.2), fulfilled if and only if $p \in\left[-2 m^{2}, 0\right]$. Thus we obtain the following.

Lemma 3.2. For an m-stage numerical method (3.11) we have

$$
\left[-l_{m}, 0\right] \subset \mathcal{S}_{m} 1
$$

where $l_{m}=2 m^{2}$. This is the optimal real negative strip that an explicit $m$-stage stochastic method can contain in its stability domain.

In what follows we show how to construct $m$-stage methods of strong order 1 with mean-square stability regions covering the mean-square stability domain $\{p, q \in$ $\mathbb{R} ; p+q^{2}<0$ and $\left.p \in\left[-c \cdot m^{2}, 0\right]\right\}$ for a given $c$ (depending on the damping parameter). In order to have a strip around the $p$-axis included in the mean-square stability region of the numerical methods, we will modify the methods (3.11) by considering damping techniques. Inspired by (3.4) we define the stochastic ROCK method with damping for $m \geq 2$ by

$$
\begin{aligned}
K_{0}= & Y_{n} \\
K_{1}= & Y_{n}+\Delta t \frac{\omega_{1}}{\omega_{0}} f\left(K_{0}\right), \\
K_{j}= & 2 \Delta t \omega_{1} \frac{T_{j-1}\left(\omega_{0}\right)}{T_{j}\left(\omega_{0}\right)} f\left(K_{j-1}\right)+2 \omega_{0} \frac{T_{j-1}\left(\omega_{0}\right)}{T_{j}\left(\omega_{0}\right)} K_{j-1}-\frac{T_{j-2}\left(\omega_{0}\right)}{T_{j}\left(\omega_{0}\right)} K_{j-2}, \\
j= & 2, \ldots, m-2, \\
K_{m-1}= & 2 \Delta t \omega_{1} \frac{T_{m-2}\left(\omega_{0}\right)}{T_{m-1}\left(\omega_{0}\right)} f\left(K_{m-2}\right)+2 \omega_{0} \frac{T_{m-2}\left(\omega_{0}\right)}{T_{m-1}\left(\omega_{0}\right)} K_{m-2}-\frac{T_{m-3}\left(\omega_{0}\right)}{T_{m-1}\left(\omega_{0}\right)} K_{m-3} \\
& +\alpha J_{n} g\left(K_{m-2}\right), \\
Y_{n+1}= & K_{m}=2 \Delta t \omega_{1} \frac{T_{m-1}\left(\omega_{0}\right)}{T_{m}\left(\omega_{0}\right)} f\left(K_{m-1}\right)+2 \omega_{0} \frac{T_{m-1}\left(\omega_{0}\right)}{T_{m}\left(\omega_{0}\right)} K_{m-1}-\frac{T_{m-2}\left(\omega_{0}\right)}{T_{m}\left(\omega_{0}\right)} K_{m-2} \\
& +J_{n}\left(\beta g\left(k_{m-2}\right)+\gamma g\left(k_{m-1}\right)\right),
\end{aligned}
$$

where

$$
\omega_{0}=1+\frac{\eta}{m^{2}}, \quad \omega_{1}=\frac{T_{m}\left(\omega_{0}\right)}{T_{m}^{\prime}\left(\omega_{0}\right)} .
$$

We observe that for $\eta=0$ these methods are similar to (3.11). Notice that 
the stability polynomials defined in (3.4) satisfy the recurrence relation

$$
\begin{aligned}
P_{j}(z) & =\frac{1}{T_{j}\left(\omega_{0}\right)} T_{j}\left(\omega_{0}+\omega_{1} z\right) \\
& =\frac{2}{T_{j}\left(\omega_{0}\right)}\left(\omega_{0}+\omega_{1} z\right) T_{j-1}\left(\omega_{0}+\omega_{1} z\right)-\frac{1}{T_{j}\left(\omega_{0}\right)} T_{j-2}\left(\omega_{0}+\omega_{1} z\right) \\
& =2 z \omega_{1} \frac{T_{j-1}\left(\omega_{0}\right)}{T_{j}\left(\omega_{0}\right)} P_{j-1}(z)+2 \omega_{0} \frac{T_{j-1}\left(\omega_{0}\right)}{T_{j}\left(\omega_{0}\right)} P_{j-1}(z)-\frac{T_{j-2}\left(\omega_{0}\right)}{T_{j}\left(\omega_{0}\right)} P_{j-2}(z) .
\end{aligned}
$$

The order of convergence of these methods is given by the following theorem.

TheOREM 3.3. For $m \geq 2$, the methods (3.18) have strong global order 1 if and only if

$$
2 \omega_{0} \alpha \frac{T_{m-1}\left(\omega_{0}\right)}{T_{m}\left(\omega_{0}\right)}+\beta+\gamma=1 \quad \text { and } \quad \alpha \gamma=\frac{1}{2}
$$

Proof. The proof can be obtained by using (3.23) and following the lines of the proof of Theorem 3.1.

We choose the set of parameters

$$
\alpha=\frac{1}{2 w_{0}} \frac{T_{m}\left(\omega_{0}\right)}{T_{m-1}\left(\omega_{0}\right)}, \quad \beta=-\frac{1}{2 \alpha}, \quad \gamma=\frac{1}{2 \alpha},
$$

which reduces to the set of parameters derived in (3.21) for methods without damping (i.e., for $\eta=0$ ). The mean-square stability function, for the above choice of $\alpha, \beta, \gamma$ and $\lambda, \mu$ real, is given by

$$
\begin{aligned}
R_{m}(p, q)= & \frac{T_{m}^{2}\left(\omega_{0}+\omega_{1} p\right)}{T_{m}^{2}\left(\omega_{0}\right)}+q^{2} \frac{T_{m}\left(\omega_{0}+\omega_{1} p\right)}{T_{m}\left(\omega_{0}\right)} \cdot \frac{T_{m-2}\left(\omega_{0}+\omega_{1} p\right)}{T_{m-2}\left(\omega_{0}\right)} \\
& +\frac{3}{4} q^{4} \frac{T_{m-2}^{2}\left(\omega_{0}+\omega_{1} p\right)}{T_{m-2}^{2}\left(\omega_{0}\right)}+q^{2}\left(\frac{T_{m-2}\left(\omega_{0}+\omega_{1} p\right)}{T_{m-2}\left(\omega_{0}\right)}\left(\frac{\omega_{1}}{\omega_{0}} p+1\right)\right. \\
& \left.+\frac{1}{2 \alpha}\left(\frac{T_{m-1}\left(\omega_{0}+\omega_{1} p\right)}{T_{m-1}\left(\omega_{0}\right)}-\frac{T_{m-2}\left(\omega_{0}+\omega_{1} p\right)}{T_{m-2}\left(\omega_{0}\right)}\right)\right)^{2} .
\end{aligned}
$$

This can be checked as for (3.13) by applying the method (3.18) to the test problem (2.10), using the recurrence relation (3.23), squaring the results, taking the expectation, and using $\mathbb{E}\left(J_{n}\right)=0, \mathbb{E}\left(J_{n}^{2}\right)=\{\Delta t\}, \mathbb{E}\left(J_{n}^{3}\right)=0$, and $\mathbb{E}\left(J_{n}^{4}\right)=3\{\Delta t\}$ (see Theorem 3.3). Let us now choose a damping parameter $\eta=0.05$ and plot the meansquare stability domains for $s=2,3,4$ in Figure 3.4. We see that compared to Figure 3.3 (where $\eta=0$ ) the stability domain along the negative real axis is now a bit shorter, but we have a strip around the negative real axis included in the stability domains. We study next how the mean-square stability domains of the methods (3.18) depend on the damping parameter. We first show that, for any fixed $\eta \geq 0$, the quadratic growth of the stability region along the negative real axis is preserved.

Proposition 3.4. Let $\eta \geq 0$. For all $m$, the $m$-stage numerical method (3.18) has a mean-square stability region $\mathcal{S}_{m}^{\eta}$ satisfying

$$
\left[-l_{m}^{\eta}, 0\right] \subset \mathcal{S}_{m}^{\eta}
$$

with $l_{m}^{\eta} \geq c(\eta) m^{2}$, and where $c(\eta)$ depends only on $\eta$.

Copyright $@$ ㅇ by SIAM. Unauthorized reproduction of this article is prohibited. 
Proof. If we set $q=0$ in (3.23), we obtain for the mean-square stability condition

$$
\left|R_{m}(p, 0)\right| \leq 1 \quad \Longleftrightarrow \quad\left|T_{m}\left(\omega_{0}+\omega_{1} p\right)\right| \leq\left|T_{m}\left(\omega_{0}\right)\right|
$$

where $\omega_{0}, \omega_{1}$ are defined in (3.19). Using well-known properties of the Chebyshev polynomials, we first observe that $T_{m}\left(\omega_{0}+\omega_{1} p\right)$ has its $m$ real zeros in the interval $D_{0}=\left[-\left(1+\omega_{0}\right) / \omega_{1},\left(1-\omega_{0}\right) / \omega_{1}\right]$. In $D_{0}$ we have $\left|T_{m}\left(\omega_{0}+\omega_{1} p\right)\right| \leq 1$, i.e., $\left|R_{m}(p, 0)\right|<$ 1. From the above stability condition, we have $R_{m}(p, 0)=1 \Longleftrightarrow T_{m}\left(\omega_{0}+\omega_{1} p\right)=$ $\pm T_{m}\left(\omega_{0}\right)$. The latter equality holds for $p=0$ and $p=-2 \omega_{0} / \omega_{1}$. The interval $D_{1}=$ $\left[-2 \omega_{0} / \omega_{1}, 0\right] \subset D_{0}$, and, since the polynomial $R_{m}(p, 0)$ is strictly monotone outside $D_{0}$, it follows that $\left|R_{m}(p, 0)\right| \leq 1 \Longleftrightarrow p \in D_{1}$, and thus $l_{m}^{\eta}=2 \omega_{0} / \omega_{1}$.

Using Taylor expansion we have

$$
T_{m}\left(\omega_{0}\right)=T_{m}\left(1+\frac{\eta}{m^{2}}\right)=\sum_{j=1}^{m} \frac{\gamma_{j}}{j !} \eta^{j}, \quad \gamma_{j}=\frac{T_{m}^{(j)}(1)}{m^{2 j}}=\prod_{i=0}^{j-1} \frac{\left(1-(i / m)^{2}\right)}{(2 i+1)} .
$$

The above sum is monotone and converges for $m \rightarrow \infty$. We define $c(\eta)=1 /\left(\sum_{j=1}^{\infty} \frac{\gamma_{j}}{j !}\right.$ $\left.\eta^{j}\right)$. By noting that $T_{m}^{\prime}\left(\omega_{0}\right) \geq m^{2}$, we obtain

$$
l_{m}^{\eta}=2 \frac{\omega_{0}}{\omega_{1}}=2\left(1+\frac{\eta}{m^{2}}\right) \frac{T_{m}{ }^{\prime}\left(\omega_{0}\right)}{T_{m}\left(\omega_{0}\right)} \geq 2\left(1+\frac{\eta}{m^{2}}\right) c(\eta) m^{2} \geq 2 c(\eta) m^{2},
$$

and the proof is complete.

We next study the limit $\eta \rightarrow \infty$. We see in the following proposition that, in this case, the growth along the negative real axis becomes linear.

Proposition 3.5.

$$
l_{m}^{\eta} \rightarrow 2 m \text { for } \eta \rightarrow \infty .
$$

Proof. Using formula (3.25) for $T_{m}\left(\omega_{0}\right), T_{m}{ }^{\prime}\left(\omega_{0}\right)$ we obtain

$$
l_{m}^{\eta}=2 m^{2}\left(\frac{1}{\eta}+\frac{1}{m^{2}}\right) \frac{\mathcal{O}\left(\frac{1}{\eta}\right)+\frac{\gamma_{m}}{(m-1) !}}{\mathcal{O}\left(\frac{1}{\eta}\right)+\frac{\gamma_{m}}{m !}}
$$

and the result follows.

Let $\mathcal{S}_{m}^{\eta}$ be the mean-square stability region of an $m$-stage method (3.18). We observe that, for increasing damping parameters $\eta$, the strip around the negative real axis included in the stability domain increases in the $q$-direction (compare Figures 3.3 and 3.4). We know from Propositions 3.4 and 3.5 that the maximum strip in the $p$-direction decreases when $\eta$ increases. Consider next the following subset of the stability domain $\mathcal{S}_{S D E}$ of the test equation (2.10):

$$
\mathcal{S}_{S D E, d}=\{(p, q) \in[-d, 0] \times \mathbb{R} ;|q| \leq \sqrt{-p}\} .
$$

The task is now to find, for a given stage number $m$, a value of $\eta$ such that $\mathcal{S}_{S D E, d_{m}^{\eta}} \subset$ $\mathcal{S}_{m}^{\eta}$, with $d_{m}^{\eta}$ as large as possible. This is done numerically and reported in Table 3.1. Such stability domains are plotted in Figure 3.5 for $m=4,7,10$. We define the class of S-ROCK methods by the numerical methods (3.18) with the optimal $d_{m}^{\eta}$ value.

In Table 3.2 we give for the S-ROCK methods listed in Table 3.1 the effective numerical work given by the ratio $m / d_{m}^{\eta}$ (work against stability).

Copyright (c) by SIAM. Unauthorized reproduction of this article is prohibited. 
TABLE 3.1

Optimal damping values for $S$-ROCK methods.

\begin{tabular}{|c|c|c|c|c|c|c|c|}
\hline$m$ & $\eta$ & $d_{m}^{\eta}$ & $l_{m}^{\eta}$ & $m$ & $\eta$ & $d_{m}^{\eta}$ & $l_{m}^{\eta}$ \\
\hline 3 & 2.2 & 5.9 & 9.6 & 5 & 12.0 & 11.2 & 13.5 \\
7 & 13.0 & 20.4 & 22.9 & 10 & 14.3 & 38.7 & 41.3 \\
25 & 20.3 & 197.6 & 200.7 & 50 & 27.2 & 679.5 & 683.2 \\
75 & 32.1 & 1405.1 & 1409.1 & 100 & 36.0 & 2358.0 & 2362.3 \\
150 & 42.1 & 4908.1 & 4912.7 & 200 & 46.7 & 8276.5 & 8281.3 \\
\hline
\end{tabular}

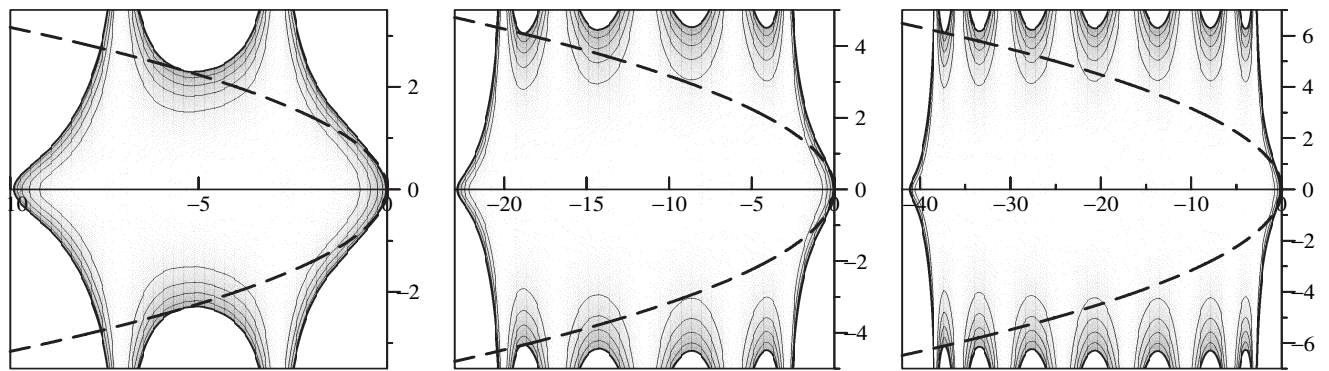

FIG. 3.5. $M S$ domain for $S$-ROCK methods with $(m=4, \eta=11.0),(m=7, \eta=13.0)$, and $(m=10, \eta=14.3)$.

TABLE 3.2

Work versus stability for $S$-ROCK methods.

\begin{tabular}{|c|c|c|c|c|c|c|c|c|c|}
\hline$m$ & $m / d_{m}^{\eta}$ & $m$ & $m / d_{m}^{\eta}$ & $m$ & $m / d_{m}^{\eta}$ & $m$ & $m / d_{m}^{\eta}$ & $m$ & $m / d_{m}^{\eta}$ \\
\hline 3 & 0.51 & 5 & 0.45 & 7 & 0.31 & 10 & 0.26 & 25 & 0.12 \\
\hline 50 & 0.07 & 75 & 0.05 & 100 & 0.04 & 150 & 0.03 & 200 & 0.02 \\
\hline
\end{tabular}

Let $\mathcal{S}_{E M}, \mathcal{S}_{P L}$, and $\mathcal{S}_{R S}$ be the stability domains of the EM, PL, and RS methods, respectively, and consider the subset of the stability domains defined in (3.29); then $\mathcal{S}_{S D E, d_{E M}} \subset \mathcal{S}_{E M}, \mathcal{S}_{S D E, d_{P L}} \subset \mathcal{S}_{P L}$, and $\mathcal{S}_{S D E, d_{R S}} \subset \mathcal{S}_{R S}$, with $d_{E M}=1, d_{P L}=31$, and $d_{R S} \simeq 0.56$ and with a work/stability ratio given by 1,2 , and 3.57 , respectively. We see the improvement obtained with the S-ROCK methods. Compared to the Platen or the RS methods (with the same order of accuracy as the S-ROCK method), we obtain a ratio up to 100 times (respectively, 179 times) smaller (for $m=200$ ). Finally let us observe that $d_{m}^{\eta} \simeq l^{\eta}$ for large $m$ and that $d_{m}^{\eta}=c(\eta) \cdot m^{2}$, where $c(\eta)$ is monotonically decreasing from 0.66 to 0.21 as $m$ increases from 3 to 200 .

Remark 3.6. Here we have restricted ourselves to S-ROCK methods with $m \leq 200$. Using the methods with a higher stage number is possible and improves the efficiency discussed above. In some numerical experiments considered in section 4 we will use $m>200$. However, due to rounding errors and internal instability, very large $m$ should be avoided [30].

Multidimensional Wiener process and weak convergence. The derivation and the order analysis of the S-ROCK methods for SDEs with multidimensional Wiener processes

$$
d Y=f(t, Y) d t+\sum_{k=1}^{M} g_{k}(t, Y) \circ d W_{k}(t), \quad Y\left(t_{0}\right)=Y_{0},
$$

Copyright $@$ by SIAM. Unauthorized reproduction of this article is prohibited. 
where $f(Y), g_{k}(Y) \in \mathbb{R}^{d}$, and $W_{k}(t)$ are independent Wiener processes, is discussed in [3]. It is well known that, in this case, the multiple stochastic integral

$$
J_{11}=\int_{t_{n}}^{t} \int_{t_{n}}^{s} \circ d W_{s 1} \circ d W_{s}
$$

cannot be expressed in a simple form using only a Wiener increment of the type $J_{k}=\int_{t_{0}}^{t_{1}} \circ d W_{k}(s)$. It is shown in [3] that the S-ROCK methods applied to (3.30) have order $1 / 2$ in the strong sense and order 1 in the weak sense. However, if the diffusion terms satisfy a commutativity condition

$$
g_{k}(t, Y(t))^{\prime} g_{r}(t, Y(t))=g_{r}(t, Y(t))^{\prime} g_{k}(t, Y(t)) \forall k, r=1, \ldots, M,
$$

then the S-ROCK methods have strong order 1 [3].

4. Numerical experiments. We give here several examples to illustrate the performance of the proposed numerical methods. We will compare the S-ROCK method with the method of Platen given in (2.3) and the RS method.

Example 1. We consider the nonlinear SDE given by

$$
d Y=\frac{\lambda}{2}\left(1-Y^{2}\right) d t+\frac{\mu}{2}\left(1-Y^{2}\right) \circ d W_{t}, \quad Y(0)=Y_{0} .
$$

This equation has an exact solution (see [20, Chap. 4.4]) given by

$$
Y(t)=\frac{\left(1+Y_{0}\right) \exp (\lambda t+\mu W(t))+Y_{0}-1}{\left(1+Y_{0}\right) \exp (\lambda t+\mu W(t))-Y_{0}+1} .
$$

Note that linearizing about the stationary solution $Y(t) \equiv-1$ leads to the test equation (2.10).

In what follows, we consider real parameters $(\lambda, \mu)$ with $\lambda$ negative. To study the mean-square stability, we choose a starting value $Y_{0}=-0.9$ close to the stationary solution. A stable solution reaches the fixed point $Y(t) \equiv-1$ after a transient (see Figure 4.1). It follows from stability results in [12, Chap. 5] (see also [16]) that the fixed point $Y(t) \equiv-1$ is mean-square stable if $\lambda+\mu^{2}<0$.

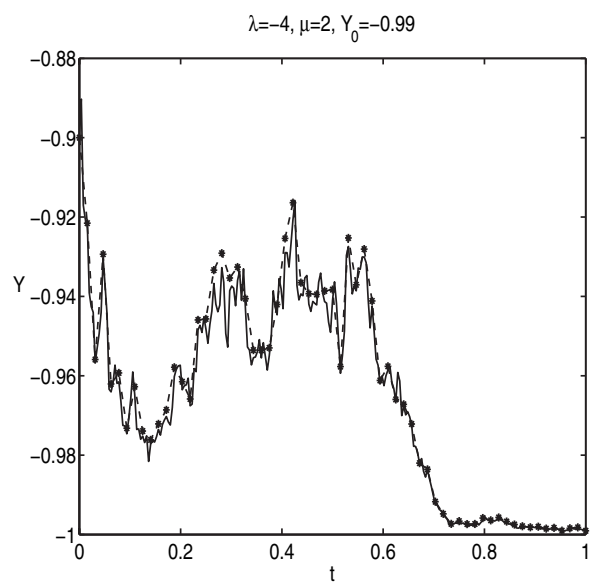

FIG. 4.1. Exact solution and numerical approximation for one Brownian path (PL method with moderately stiff parameters).

Copyright (c) by SIAM. Unauthorized reproduction of this article is prohibited. 


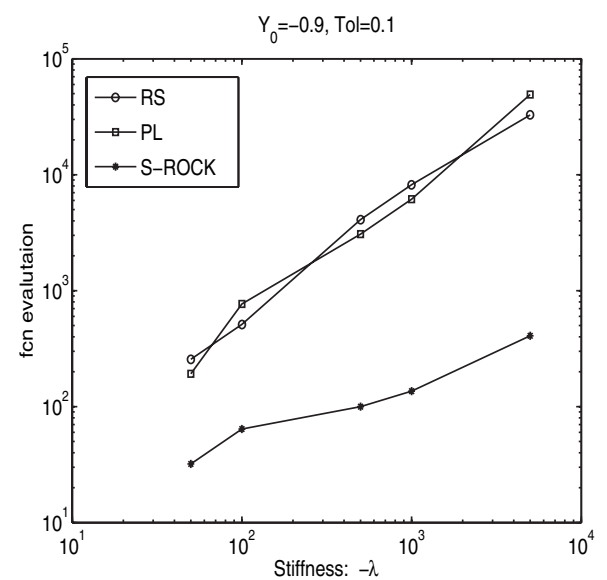

FIG. 4.2. Stiffness versus function evaluations for $R S, P L$, and $S$-ROCK (with stage number $m=6,9,23,32,100$.

We solve numerically (4.1) with the three methods PL, RS, and S-ROCK over $0 \leq t \leq 1$. To estimate the error in the strong sense at time $T=1$ for given step size $\Delta t$, we choose $n$ such that $n \Delta t=1$ and approximate

$$
e_{\Delta t}^{\text {strong }}:=\mathbb{E}\left|y_{n}-y(T)\right| \leq T o l,
$$

by averaging the endpoint error over $N=10^{5}$ numerically generated paths. The sampling error, which is known to decay as $1 / \sqrt{N}$, is negligible here.

We fix a tolerance $T o l=10^{-1}$, vary the values of $\lambda$ and $\mu$, and determine the step size (chosen as $\Delta t=2^{-p}$ ) for PL and RS so that the required precision is obtained. We know that the linearized solution about $Y(t) \equiv-1$ is stable if and only if $\Re \lambda+\Re \mu^{2}<0$. We choose the real parameters $(\lambda, \mu)$ close to the boundary of the stability region $\mu=$ $\sqrt{-\lambda-1}$ and increase the stiffness by choosing $\lambda=-50,-100,-500,-1000,-5000$. For the S-ROCK methods, we chose the step size to obtain the required accuracy and a stage number so that the numerical solution is stable.

We see in Figure 4.2 that the S-ROCK methods perform significantly better than the PL or the RS method as the stiffness increases. At the maximum stiffness $\left(\lambda=-5000\right.$ ), the step size of $\Delta t=2^{-13}$ and $\Delta t=2^{-14}$ has to be chosen (for stability reasons) for the PL and RS, to solve (4.1), whereas a step size of $\Delta t=2^{-2}$ can be chosen for the S-ROCK method. Note that for PL and RS the step sizes, determined by the stability requirement, are much smaller than needed to obtain the desired accuracy. As a consequence, the errors are always much smaller than the given tolerance. For the S-ROCK method, a step size governed by the accuracy requirement can be chosen to solve this problem.

Example 2. For the second example we consider a stochastic partial differential equation, the heat equation with noise, given by

$$
d u(t, x)=(D \Delta u(t, x)) d t+k u(t, x) \circ d W_{t},
$$

where $(t, x) \in[0, T] \times[0,1], D$ is the diffusion coefficient, and $k$ is a noise parameter. We choose $u(0, x)=1$ as the initial condition, and mixed boundary conditions are given by $u(t, 0)=5,\left.\frac{\partial u(t, x)}{\partial x}\right|_{x=1}=0$. For simplicity we set $D=k=1$ in what follows. 


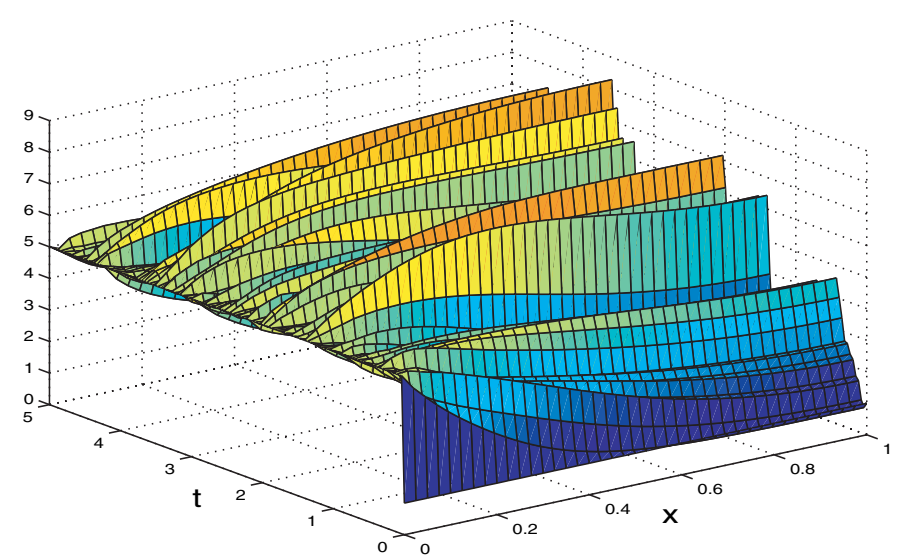

FIG. 4.3. Numerical solution of the problem (4.6) with the PL method (one trajectory, $N=40$ ).

We discretize the space interval in $N+1$ equidistant points $x_{i}=0, \ldots, N$, with $h=x_{i+1}-x_{i}=1 / N, x_{0}=0, x_{N}=1$, and obtain a system of $N$ SDEs given by

$$
d Y_{t}^{i}=\frac{Y_{t}^{i+1}-2 Y_{t}^{i}+Y_{t}^{i-1}}{h^{2}}+Y_{t}^{i} \circ d W_{t}, j=1, \ldots, N
$$

where $Y_{t}^{0}=5, Y_{t}^{N+1}=Y_{t}^{N-1}$ are given by the boundary conditions. The deterministic part of the linear system above is known to have eigenvalues distributed around the negative real axis in the interval $\left(-4 / h^{2}, 0\right)$. Thus, a necessary condition for the stability of a numerical method is $\Delta t \cdot\left(4 / h^{2}\right) \in \mathcal{S}$. This condition is well known to be prohibitively restrictive as $N$ increases or, equivalently, as the spatial discretization is refined. Equation (4.5) can be rewritten as

$$
d Y_{t}=(A Y+B) d t+k Y \circ d W_{t},
$$

where $Y=\left(Y^{1}, \ldots, Y^{N-1}\right)^{T}, B=\left(5 / h^{2}, 0, \ldots, 0\right)^{T}$, with initial conditions given by $Y_{0}=(1, \ldots, 1)^{T}$ and

$$
A=\frac{1}{h^{2}}\left(\begin{array}{ccccc}
-2 & 1 & & & \\
1 & -2 & 1 & & \\
& \ddots & \ddots & \ddots & \\
& & 1 & -2 & 1 \\
& & & 2 & -1
\end{array}\right)
$$

The exact solution of (4.6) is given by $Y(t)=\Phi(t) Y_{0}+\Phi(t) \int_{0}^{t} \Phi(s)^{-1} B d s$, where

$$
\Phi(t)=\exp (A t+I W(t))
$$

where $I$ is the $N \times N$ identity matrix (see [4, Chap. 8] for details). We solve numerically (4.6) with the three methods PL, RS, and S-ROCK over $0 \leq t \leq 5$ for various values of $N=40,100,500$, thus increasing the stiffness. We sketched in Figure 4.3 one trajectory of a numerical solution.

For the numerical comparison, we choose an initial integration step $\Delta t=0.5 \cdot 10^{-6}$ to have a theoretical strong error smaller than $<10^{-1}$. As in the previous example, 
TABLE 4.1

Function evaluations for the stable integration of the problem (4.6) (value for one trajectory).

\begin{tabular}{|c|c|c|c|}
\hline & $N=40$ & $N=100$ & $N=500$ \\
\hline PL & $4.9152 \cdot 10^{4}$ & $3.93216 \cdot 10^{5}$ & $1.2582912 \cdot 10^{7}$ \\
\hline RS & $3.2768 \cdot 10^{4}$ & $2.62144 \cdot 10^{5}$ & $8.388608 \cdot 10^{6}$ \\
\hline S-ROCK & $2.816 \cdot 10^{3}, s=42$ & $7.616 \cdot 10^{3}, s=117$ & $8.2432 \cdot 10^{4}, s=320$ \\
\hline
\end{tabular}

TABLE 4.2

Step size for the stable integration of the problem (4.6).

\begin{tabular}{|c|c|c|c|}
\hline & $N=40$ & $N=100$ & $N=500$ \\
\hline $\mathrm{PL}$ & $5 \cdot 2^{-14}$ & $5 \cdot 2^{-17}$ & $5 \cdot 2^{-22}$ \\
\hline $\mathrm{RS}$ & $5 \cdot 2^{-13}$ & $5 \cdot 2^{-16}$ & $5 \cdot 2^{-21}$ \\
\hline S-ROCK & $5 \cdot 2^{-6}$ & $5 \cdot 2^{-6}$ & $5 \cdot 2^{-8}$ \\
\hline
\end{tabular}

we estimate the error $e_{\Delta t}^{\text {strong }}$ by averaging the endpoint error over $10^{4}$ realizations. For the PL and RS methods, we always run into instability, i.e., $e_{\Delta t}^{\text {strong }}>>1$ for the aforementioned initial step size and for the chosen spatial discretization. We thus decrease the step size, chosen here as $\Delta t=0.5 \cdot 2^{-r}, r=6,7, \ldots$, until $e_{\Delta t}^{\text {strong }} \leq 10^{-1}$. For the S-ROCK methods, the initial step size ensures $e_{\Delta t}^{\text {strong }} \leq 10^{-1}$ provided that the stage number is adapted. For the numerical experiments with $N=500$ the value of $m$ to have a stable integration with $\Delta t=0.5 \cdot 10^{-6}$ is quite large $(m>500)$, and we therefore reduced the step size to $\Delta t=0.5 \cdot 10^{-8}$ (see Remark 3.6). For various $N$, we report in Table 4.1 the number of function evaluations and in Table 4.2 the corresponding step sizes needed to have a stable numerical solution.

We see for the PL and RS methods, as in Example 1, that the step sizes are determined by the stability requirement and are much smaller than predicted by the order of convergence of the methods. This behavior for classical explicit methods is expected for stiff problems. Since the diffusion term is small compared to the drift term, we also see that the RS method performs better. This can be explained as follows. For the RS method, the stability domain along the $p$-axis is twice as big as the corresponding region for the PL method, while the number of function evaluations per step is $4 / 3$ times the number of function evaluations of the PL method. Finally, we see the tremendous improvement obtained with the S-ROCK method as the spatial discretization is refined.

Acknowledgments. The authors are grateful to Ernst Hairer and Gerhard Wanner for helpful discussions and to Weinan E and Des Higham for useful comments on the draft of this paper.

\section{REFERENCES}

[1] A. Abdulle And A. A. Medovikov, Second order Chebyshev methods based on orthogonal polynomials, Numer. Math., 90 (2001), pp. 1-18.

[2] A. AbDulle, Fourth order Chebyshev methods with recurrence relation, SIAM J. Sci. Comput., 23 (2002), pp. 2041-2054.

[3] A. Abdulle And S. Cirilli, Stabilized methods for stiff stochastic systems, C. R. Acad. Sci. Paris Ser. I, 345 (2007), pp. 593-598.

[4] L. Arnold, Stochastic Differential Equation, Theory and Application, Wiley, New York, 1974.

[5] K. Burrage and P. M. Burrage, Order conditions of stochastic Runge-Kutta methods by $B$-series, SIAM J. Numer. Anal., 38 (2000), pp. 1626-1646. 
[6] K. Burrage, P. M. Burrage, and T. Tian, Numerical methods for strong solutions of SDES, Proc. R. Soc. Lond., 460 (2004), pp. 373-402.

[7] P. M. Burrage, Runge-Kutta Methods for Stochastic Differential Equations, Ph.D. thesis, University of Queensland, Brisbane, Australia, 1999.

[8] S. Cirilli, Méthodes de Chebyshev stochastiques et équations aux dérivées partielles stochastiques, thèse 3349, Université de Genève, 2002.

[9] W. E, Analysis of the heterogeneous multiscale method for ordinary differential equations, Commun. Math. Sci., 1 (2003), pp. 423-436.

[10] W. E, D. Liu, And E. Vanden-Eijnden, Analysis of multiscale methods for stochastic differential equations, Comm. Pure Appl. Math., 2004, pp. 1-48.

[11] K. Eriksson, C. Johnson, And A. LoGG, Explicit time-stepping for stiff ODEs, SIAM J. Sci. Comput., 25 (2003), pp. 1142-1157.

[12] T. C. GARD, Introduction to Stochastic Differential Equations, Marcel Dekker, New York, 1988.

[13] C. W. Gear and I. G. Kevrekidis, Projective methods for stiff differential equations: Problems with gaps in their eigenvalue spectrum, SIAM J. Sci. Comput., 24 (2003), pp. 10911106.

[14] A. Guillou And B. Lago, Domaine de stabilité associé aux formules d'intégration numérique d'équations différentielles, à pas séparés et à pas liés. Recherche de formules à grand rayon de stabilité, in Proceedings of the 1er Congr. Assoc. Fran. Calcul (AFCAL), Grenoble, 1960, pp. 43-56.

[15] E. Hairer and G. Wanner, Solving Ordinary Differential Equations II. Stiff and DifferentialAlgebraic Problems, 2nd ed., Springer-Verlag, Berlin, 1996.

[16] R. Z. Has'minskil̆, Stochastic Stability of Differential Equations, Sijthoff \& Noordhoff, Groningen, The Netherlands, 1980.

[17] D. J. Higham, A-stability and stochastic mean-square stability, BIT, 40 (2000), pp. 404-409.

[18] D. J. Higham, Mean-square and asymptotic stability of the stochastic theta method, SIAM J. Numer. Anal., 38 (2000), pp. 753-769.

[19] P. J. VAn Der Houwen ANd B. P. Sommeijer, On the internal stage Runge-Kutta methods for large m-values, Z. Angew. Math. Mech., 60 (1980), pp. 479-485.

[20] P. E. Kloeden and E. Platen, Numerical Solution of Stochastic Differential Equations, Springer-Verlag, Berlin, 1992.

[21] V. I. Lebedev, How to Solve Stiff Systems of Differential Equations by Explicit Methods, CRC Press, Boca Raton, FL, 1994, pp. 45-80.

[22] G. Maruyama, Continuous Markov processes and stochastic equations, Rend. Circ. Mat. Palermo, 4 (1955), pp. 48-90.

[23] G. N. Milstein, Approximate integration of stochastic differential equations, Theory Probab. Appl., 19 (1974), pp. 557-562.

[24] G. N. Milstein and M. V. Tretyakov, Stochastic Numerics for Mathematical Physics, Scientific Computing, Springer-Verlag, New York, 2004.

[25] G. N. Milstein, E. Platen, and H. Schurz, Balanced implicit methods for stiff stochastic systems, SIAM J. Numer. Anal., 35 (1998), pp. 1010-1019.

[26] B. Oksendal, Stochastic Differential Equations, 6th ed., Springer-Verlag, Berlin, 2005.

[27] E. Platen, Zur zeitdiskreten approximation von Itoprozessen, Diss. B. Imath. Akad. des Wiss. der DDR, Berlin, 1984.

[28] A. Rodkina AND H. Schurz, Almost sure asymptotic stability of drift-implicit $\theta$-methods for bilinear ordinary stochastic differential equations in $\mathbb{R}^{1}$, J. Comput. Appl. Math., 180 (2005), pp. 12-31.

[29] Y. Saito and T. Mitsui, Stability analysis of numerical schemes for stochastic differential equations, SIAM J. Numer. Anal., 33 (1996), pp. 2254-2267.

[30] B. P. Sommeijer, L. F. Shampine, and J. G. Verwer, RKC: An explicit solver for parabolic PDEs, J. Comput. Appl. Math., 88 (1998), pp. 316-326.

[31] E. VAnden-Eijnden, Numerical techniques for multiscale dynamical system with stochastic effects, Commun. Math. Sci., 1 (2003), pp. 385-391.

Copyright (c) by SIAM. Unauthorized reproduction of this article is prohibited. 